When God Stops Fighting 



\section{When God Stops Fighting}

HOW RELIGIOUS VIOLENCE ENDS

Mark Juergensmeyer

UNIVERSITY OF CALIFORNIA PRESS 
University of California Press

Oakland, California

(C) 2022 by Mark Juergensmeyer

Library of Congress Cataloging-in-Publication Data

Names: Juergensmeyer, Mark, author.

Title: When God stops fighting : how religious violence ends / Mark Juergensmeyer.

Description: [Oakland, California] : [University of California Press], [2022] | Includes bibliographical references and index..

Identifiers: LC C N 2021021120 (print) | LC C N 2021021121 (ebook) | IS BN 9780520384729 (hardcover) | IS BN 9780520384736 (paperback) | ISBN 9780520384743 (ebook)

Subjects: LCSH: Violence-Religious aspects-Islam. | Violence-IraqReligious aspects. | Violence-Philippines-Religious aspects. |

Violence-India-Punjab-Religious aspects. | Religious militantsInterviews. | BISAC: RELIGION / Religion, Politics \& State | SOCIAL

SCIENCE / Violence in Society

Classification: LCC BP190.5.v56 J84 2022 (print) |

LCC BP190.5.v56 (ebook) | DDC 297.2/7-dc23

LC record available at https://lccn.loc.gov/2021021120

LC ebook record available at https://lccn.loc.gov/2021021121

Manufactured in the United States of America

$\begin{array}{lllll}25 & 24 & 23 & 22 & 21\end{array}$

$\begin{array}{llllllllll}10 & 9 & 8 & 7 & 6 & 5 & 4 & 3 & 2 & 1\end{array}$ 